\title{
Impact of a Novel Approach for Undergraduate Medical Ethics Education in The Middle East and South Asia
}

Muhammad Shahid Shamim ( $\sim$ doctsaab@gmail.com )

Dow University of Health Sciences

Lubna Baig

APPNA Institute of Public Health Jinnah Sindh Medical University

Adrienne Torda

University of New South Wales

Chinthaka Balasooriya

University of New South Wales

\section{Research Article}

Keywords: Medical Education, Ethics Education, Educational Strategy, Workbook

Posted Date: August 19th, 2021

DOI: https://doi.org/10.21203/rs.3.rs-658417/v1

License: (a) (i) This work is licensed under a Creative Commons Attribution 4.0 International License.

Read Full License 


\section{Abstract \\ Background}

Complexities of ethics education, combined with socio-cultural issues specific to the medical curricula of the developing country context, have created resource constraints that impact medical ethics education delivery in countries like Pakistan and Saudi Arabia. These constraints include the dearth of contextually relevant texts, shortage of trained teachers and confines in institutional regulations in the regions. Focused strategies are required to address these constraints. Workbook-based ethics learning (WBEL) is one such strategy based on a contextually relevant ethics education model.

\section{Purpose}

This study aimed to evaluate the effectiveness of ethics education, delivered through the WBEL strategy, on students' learning and satisfaction in medical colleges of Pakistan and Saudi Arabia.

\section{Methods}

A pre-post-test intervention study was conducted in two medical colleges, Jinnah Sindh Medical University, Pakistan and King Abdulaziz University, Saudi Arabia. An ethics course was structured using the WBEL strategy and delivered to early clinical years medical students. The impact on student learning was measured by pre-and-post administration of a set of Key Feature Questions (KFQ) and a Script Concordance Test (SCT). A twenty-eight-item course feedback questionnaire was developed to collect students' perceptions regarding the WBEL strategy.

\section{Results}

Of the total 125 students who consented, 90 (72\%) completed the pre-and post-test measures, and 103 returned the feedback forms at the two sites. The post-test scores in KFQs were significantly higher than pretest scores in both Pakistani and Saudi students ( $p$-value $<0.001)$. There was no significant pre-to-post difference in overall SCT scores $(p=0.057)$. The majority of participants considered the overall course to be valuable. They provided positive feedback on the Workbook's usefulness and the enhancement of critical thinking within the course. Ethics case discussions, classroom quizzes, and reflective writing were considered most effective for learning during the course.

\section{Conclusion}

The findings suggest that the WBEL is a promising way forward in medical ethics education as it resulted in a notable increase in participants' knowledge and ethical decision-making skills. The study also 
provides evidence of feasibility for using the WBEL strategy for teaching ethics in resource-limited regions.

\section{Background}

Competency in medical ethics is considered essential for medical graduates [1, 2]. It is reflected in the goals of formal curricula in almost all healthcare institutes and medical programs [3-5]. Achieving this goal has remained a complex task for educators around the World [6-9]. Additionally, the influences of the socio-cultural environment of a region or country further add to the complexities of this discipline [4, $10,11]$. The implications of this can be especially problematic for medical curricula in countries like Pakistan and Saudi Arabia, representing the Middle East and South Asian regions, which share features that make them different from most developed western cultures. These countries have several common resource constraints in relation to undergraduate medical ethics education [12]. These include nonavailability of culturally relevant texts, a limited number of trained medical ethics teachers and institutional policies which do not suppport the implementation of ethics as an essential part of the curriculum [13]. To develop a successful approach to ethics education in the face of such constraints, a thoughtfully designed and contextually tailored educational approach is required [14-16].

An innovative educational strategy, named workbook-based ethics learning (WBEL), was developed using a robust consultative process undertaken in a prior study [17]. This innovative strategy is grounded in the principles of cognitive load theory and contextually relevant ethics education model (CREEM, Fig. 1).

The model is considered useful to scaffold the process of ethics education to provide culturally relevant learning experiences, safe and respectful environments for ethical discourse and opportunities for reflection and feedback [18]. The development and refinement of the WBEL through the consultative process [17] ensured that the strategy has the characteristics required for the effective delivery of ethics education. With these characteristics, the WBEL strategy has the potential to 1) be modified to suit the contextually relevant needs of the society where it is being implemented, 2) address the resource constraints to medical ethics education by providing contextual reading material and guidance to the teachers on effective delivery of ethics education, and 3) provide engaging exercises for social-learning activities, and structure for reflection and feedback, while ensuring that the cognitive load remains appropriate.

The purpose of the current study was to evaluate the effectiveness of the WBEL strategy on students' knowledge and ethical decision-making skills and engagement in the context of Pakistan and Saudi Arabia. This research's implications may contribute to the development of ethics education in other regions with similar context.

\section{Methods}


The ethics approval for this study was acquired from the ethics review committee of King Abdulaziz University (reference no. 393 - 15), Jinnah Sindh Medical University (reference no. 2016-30), where the study was conducted, and the University of New South Wales (UNSW) (approval no. HC15640) Australia, where the primary author was enrolled as a PhD scholar. All methods were performed in accordance with the relevant guidelines and regulations of UNSW.

\section{Study design and sample size}

This study employed a Quasi-experimental, pre-post-test intervention design. All the study participants received the intervention, the EWB, in an ethics course delivered through the WBEL strategy, and the study measured students' progress across and within the participating groups.

An a priori power analysis sample size calculation was performed using $\mathrm{G}^{*}$ Power, version 3 , commonly used in the social, behavioural, and biomedical sciences [19]. The magnitude of the effect size was interpreted on Cohen's suggestions of a d of 0.30 as "small", $d$ of 0.50 as "medium", and d of 0.80 as "large" [20]. A value between medium and large was estimated based on previous studies that used Script Concordance Test (SCT) [21-23] and Key Feature Questions (KFQ) [24] for assessing clinical reasoning in various situations, and discussion with the experts. The calculation revealed that an adequate sample size of 64 students has $90 \%$ power to detect an effect size of $d=0.6$ with an alpha of 0.05 (two sides).

\section{Participant recruitment}

The ethics course was delivered to early clinical year students at the two medical schools: fourth-year students in a six-year program at Rabigh Faculty of Medicine at KAU in September 2017, and third-year students in a five-year program at Sindh Medical College at JSMU in March 2018.

The students attending the course in their respective settings were informed about the study and invited to participate by a non-faculty staff member. The courses' delivery was kept as similar in the two locations as possible, including the same primary facilitator. The course format included short, interactive lectures, video demonstrations, role-plays, case and topic discussions.

The pretest was conducted at the beginning, on the first day of the course. The students were asked to complete the feedback forms and the post-test at the end of the course. All the collected data were deidentified before analysis.

\section{The intervention}

The ethics course designed using the WBEL strategy and spanning over 30 contact hours [25], structured using the WBEL strategy, was delivered in undergraduate medical programs at the two sites. This strategy used a workbook specifically designed through a robust consultative process [17] to enhance the delivery of the ethics content. The Workbook was designed as a complete resource to address the constraints introduced above. Figure 2 shows the content of the Workbook. 
The content in the Workbook was sequenced to optimise the cognitive load for students [26, 27]. The initial part of the Workbook introduced users to course outcomes, teaching-learning methods used within the course, and a description of how to use the Workbook during the course. Each topic was introduced with some short reading material which provided an overview of the content in clear, easy to understand language to activate students' working memory. Specific learning outcomes for the topics (Fig. 3) and relevant material from national guides and codes of ethics were incorporated within the Workbook, along with the description of topics, where needed, in coloured boxes to make it user-friendly and to decrease the extrinsic load [28]. The reading was followed by interactive activities for students, designed as constructive learning experiences, with observation and reflection. Such constructive learning activities have shown to enhance students' learning [29]. The activities included case discussion vignettes, roleplays and video clips to initiate discussions with peers and provide a basis for collaborative, constructive learning [30]. Space for documenting students' reflections and faculty feedback is provided after every Workbook activity (Fig. 4).

Our main objective was to develop students' ability to identify and resolve potential ethical issues in their context and discuss contemporary ethical issues faced by physicians worldwide.

Each of the student attending the course received the Workbook to be used in the WBEL process. They used the Workbook to complete the following stages of learning (Fig. 1), derived from the CREEM [18]:

- Step 1: The Learning experience

The learning was triggered by an experience designed within the students' socio-cultural context. A case vignette, role-play or video clip with an ethical issue in healthcare delivery is used to trigger the learning process.

- Step 2: Assimilation, refinement and organisation

The student explores background information relevant to the ethical issues through the reading material in the Workbook to identify the information relevant to analyse the given context.

- Step 3: Experimentation, accommodation and consolidation

The learning process moves forward when the student tries to make sense of the new concepts by using the information to address the ethical issues, discuss what $s /$ he has learned to enhance understanding of the issues within the context and make informed decisions to resolve the relevant issues.

The learning process is supported by the students' social interactions with peers and facilitator during the discourse. The students reflect on their newly gained experience and receive feedback from the facilitator, which further refines their understanding.

\section{Evaluation tools}


Pre-and post-measures evaluated the effectiveness of the intervention in this study. Learners' views on the intervention were collected via a survey form. These measurement tools and the survey instrument are described below.

\section{Pre-post-test measures:}

The WBEL strategy's effectiveness was evaluated using pre-and post-test administration of a Script Concordance Test (SCT) and Key Feature Questions (KFQ) specifically designed for this research. SCTs have been shown to assess students' ethical reasoning skills in situations with uncertainty [31]. They are highly suitable in situations where there can be more than one correct answer [32]. The SCTs in this study provided a means to assess students' decision-making abilities in relation to the given scenarios. They assessed students' ability to analyse their decisions when new information was introduced.

KFQ focuses on challenging aspects of clinical problems where students are more likely to make errors [33]. They are based on problem-solving in clinical case scenarios. In a typical KFQ, the case briefly describes a problem with a critical feature embedded in the description. The key feature forms the stem and is followed by one or more questions. The questions are aimed at key features or problems in the clinical case rather than factual knowledge. The learners are required to make relevant decisions by identifying the key features in the case [34]. KFQs have been used in various formats, including MCQ, short answer and True/False formats, in paper-pencil and computerised versions [35]. The SCT and KFQ items were developed explicitly for this study by one of the authors (MSS), following the recommendations given by Fournier and colleagues [36] and Lubarsky and colleagues [37]. The items were reviewed independently by the research team members (CB and AT) and four experienced ethics educators in the region to ensure relevance, content and face validity [38]. Twenty SCTs with three questions per vignette and ten KFQs with two questions in each were included in the final version of the test paper. Testing time of 90 minutes was considered adequate for this evaluation in this context [39]. Test takers were provided with a 30-minute orientation before the assessment, in which they were informed about the assessment process and tools.

\section{Students' feedback:}

Written anonymous feedback from students was gathered to evaluate their satisfaction, impact on learning and feasibility of the intervention. This was done using the Course Feedback Questionnaire (CFQ), constructed by adapting two instruments:

- The Motivated Strategies for Learning Questionnaire (MSLQ), developed at the University of Michigan [40]. The original version of MSLQ consists of 81 items distributed across 15 scales. Each item is scored on a seven-point Likert-type measure. MSLQ is extensively used for theoretical and applied studies in diverse samples and settings [41]. Two subscales of MSLQ were found to be relevant for the evaluation of the WBL strategy in this research. These included six items of the Task value scale and five items of the Critical thinking scale. 
- Feedback Questionnaire 2 (FQ2) was developed for a previous study conducted in the same context [42]. The FQ2 included a total of 27 items which were piloted with previous year students in the study setting. Based on the experience gained from the pilot, the items were reviewed and modified for comprehensibility, feasibility and presentation. Seventeen items were finally selected from FQ2 for incorporation in the CFQ developed for this study. These items included three questions regarding the use of a workbook and 14 on various teaching methods employed to facilitate learning during the course.

Thereby, the CFQ in this research consisted of 28 items (Table 1), including eleven items from the MSLQ and 17 items from FQ2. The research team then reviewed the 28 items CFQ to establish face and content validity [43]. 
Course feedback questionnaire statements

\section{What I have learned in this course will help me in other courses (MSLQ 4)}

$2 \quad$ I liked using the Workbook for learning during the course

3 It is important for me to learn the material in this course (MSLQ 10)

$4 \quad$ I enjoyed the ethics and professionalism course

$5 \quad$ I am very interested in the content given in the Workbook (MSLQ 17)

6 This course will help me in resolving ethical problems in my professional life

7 I think the Workbook was useful for my learning (MSLQ 23)

8 The reading material given in the workbook was very helpful for my learning in this course

$9 \quad$ l liked the subject matter of this course (MSLQ 26)

10 Understanding the subject matter of this course was very important for me (MSLQ 27)

11 I found the classroom quizzes very useful for my learning in this course

12 I often questioned things I read in the workbook to decide if I find them convincing (MSLQ 38)

13 The ethical case discussions in the classroom was very useful for my learning in this course

14 When a theory, interpretation, or conclusion is presented in class or in the Workbook, I try to decide if there is good supporting evidence (MSLQ 47)

15 Reflective writing in Workbook was very useful for my learning in this course

16 The student presentations in the classroom were very useful for my learning in this course

17 I treat the course material as a starting point and try to develop my own ideas about it (MSLQ 51)

18 I found the movie clips and videos very useful for my learning in this course

19 I try to play around with ideas of my own related to what I have learned in this course (MSLQ 66)

20 I learned a lot from people sharing their experiences during the course

21 Whenever I read or heard a statement or conclusion in this class, I thought about possible alternatives (MSLQ 71)

22 Rate the usefulness of each of the following activities for your learning during the course
a) Classroom quizzes
b) Ethics case discussions
c) Experience sharing
d) Movie clips/Video demonstrations 


\section{What I have learned in this course will help me in other courses (MSLQ 4)}

e) Reflective writing

f) Student presentation

g) Role-plays

\section{Analysis}

All the data was entered and analysed on SPSS version 24. Frequencies and percentages were calculated for gender, location, and feedback domains.

Inference on the impact of the intervention on learning was drawn by statistically analysing the improvement in scores on SCT and KFQ items in the pre-post-test measure. Mean \pm SD or Median (IQR) were calculated for all SCT and KFQ questions. Independent sample $t$-test and Mann Whitney Utest were applied to assess the significant difference of SCT and KFQ scores between various groups. Paired $t$-test was applied to assess pre-and post-training scores on SCT and KFQ.

The internal consistency reliability using Cronbach's alpha of the CFQ was initially calculated for investigating its appropriateness. The analysis of scores from the 7-point Likert scale on CFQ was performed according to the guidance given for MSLQ [44]. Chi-square test and Fisher exact test were applied to assess the significant association between categorical variables and feedback domains.

A p-value of less than 0.05 was considered statistically significant.

\section{Results}

The overall pretest scores between JSMU and KAU students did not show statistically significant differences, suggesting that the two cohorts were similar. Therefore, the data from cohorts were considered together for measuring the impact of the intervention.

Table 2 shows participants from the two universities. Rabigh Faculty of Medicine at KAU was an all-men institute at the time of the study, while at JSMU, female students were significantly more in number. Overall, the study sample was evenly distributed between male (42\%) and female (58\%) students; however, there was a gender imbalance between the two locations where the study was conducted. 
Table 2

Participation of students

\begin{tabular}{|llll|}
\hline & Number of students & & \\
\cline { 2 - 4 } & JSMU & KAU & Total \\
\hline Attended ethics course & 88 & 37 & 125 \\
\hline Completed pre- \& post- test for analysis & 63 & 27 & 90 \\
& (male 10, female 53) & (all male) & (male 37: female 53) \\
\hline Feedback forms returned & 72 & 31 & 103 \\
& (male 12, female 60) & (all male) & (male 43: female 60) \\
\hline
\end{tabular}

Of the total 125 students who attended the ethics course at the two sites, $90(72 \%)$ completed the pre-and post-test measures, and 103 (82\%) returned the CFQ feedback forms. The post-test scores in KFQs were significantly higher as compared to pretest scores ( $p$-value $<0.001)$. There was no significant difference in overall SCT scores $(p=0.057)$ between pre-and post-test (Table 3$)$. When SCT items were analysed individually, significant improvement was noted in 14 out of 60 items (Table 3). The McNemar test was used to explore the difference between their knowledge and skills "before and after" design. These were found significant in 10 of 60 SCT items (Table 4) and 12 of $20 \mathrm{KFQ}$ items (Table 5). 
Table 3

Pre- and post- test comparisons

\begin{tabular}{|c|c|c|c|c|c|c|c|c|}
\hline \multirow[t]{2}{*}{ Questions } & \multirow[t]{2}{*}{$\mathbf{N}$} & \multicolumn{3}{|c|}{ Pre- test } & \multicolumn{3}{|c|}{ Post- test } & \multirow[t]{2}{*}{$P$ value } \\
\hline & & $\begin{array}{l}\text { Mean } \\
\pm \text { SD }\end{array}$ & $\begin{array}{l}\text { Min- } \\
\text { Max }\end{array}$ & Median(IQR) & $\begin{array}{l}\text { Mean } \\
\pm \text { SD }\end{array}$ & $\begin{array}{l}\text { Min- } \\
\text { Max }\end{array}$ & Median(IQR) & \\
\hline \multicolumn{9}{|l|}{ Pakistan } \\
\hline SCT & 63 & $\begin{array}{l}16 \pm \\
2.9\end{array}$ & $\begin{array}{l}8.9- \\
20.6\end{array}$ & - & $\begin{array}{l}16.7 \pm \\
2.8\end{array}$ & $\begin{array}{l}10.8- \\
21.8\end{array}$ & - & $0.075^{\rrbracket}$ \\
\hline KFQ & 63 & - & $0-16$ & $8(6-11)$ & - & $0-19$ & $12(10-14)$ & $0.000^{\star \star \otimes}$ \\
\hline \multicolumn{9}{|l|}{ Saudia } \\
\hline SCT & 27 & $\begin{array}{l}14.9 \pm \\
2.8\end{array}$ & $\begin{array}{l}9.5- \\
22\end{array}$ & - & $\begin{array}{l}15.3 \pm \\
3\end{array}$ & $\begin{array}{l}10.5- \\
21.1\end{array}$ & - & $0.075^{\rrbracket}$ \\
\hline KFQ & 27 & $\begin{array}{l}6.2 \pm \\
2.5\end{array}$ & $0-13$ & - & $9.6 \pm 4$ & $2-17$ & - & $0.000^{* \star \mathbb{X}}$ \\
\hline \multicolumn{9}{|l|}{ Female } \\
\hline SCT & 53 & $\begin{array}{l}16 \pm \\
2.9\end{array}$ & $\begin{array}{l}8.9- \\
20.6\end{array}$ & - & $\begin{array}{l}16.7 \pm \\
2.7\end{array}$ & $\begin{array}{l}10.8- \\
21.8\end{array}$ & - & $0.075^{\rrbracket}$ \\
\hline KFQ & 53 & - & $0-16$ & $8(6-11)$ & - & $0-19$ & $12(10-14)$ & $0.000^{\star \star \mathbb{Z}}$ \\
\hline \multicolumn{9}{|l|}{ Male } \\
\hline SCT & 37 & $\begin{array}{l}15.2 \pm \\
2.9\end{array}$ & $\begin{array}{l}9.5- \\
22\end{array}$ & - & $\begin{array}{l}15.6 \pm \\
3.1\end{array}$ & $\begin{array}{l}10.5- \\
21.1\end{array}$ & - & $0.000^{\star \star \mathbb{}}$ \\
\hline KFQ & 37 & $\begin{array}{l}6.7 \pm \\
2.7\end{array}$ & $0-13$ & - & $\begin{array}{l}9.9 \pm \\
4.3\end{array}$ & $0-18$ & - & $0.015^{\star \bigotimes}$ \\
\hline \multicolumn{9}{|l|}{ Overall } \\
\hline SCT & 90 & $\begin{array}{l}15.7 \pm \\
2.9\end{array}$ & $\begin{array}{l}8.9- \\
22\end{array}$ & - & $\begin{array}{l}16.3 \pm \\
2.9\end{array}$ & $\begin{array}{l}10.5- \\
21.8\end{array}$ & - & $0.057^{\rrbracket}$ \\
\hline KFQ & 90 & $\begin{array}{l}7.4 \pm \\
3.8\end{array}$ & $0-16$ & - & $\begin{array}{l}11.1 \pm \\
3.9\end{array}$ & $0-19$ & - & $0.000^{\star \star \mathbb{Q}}$ \\
\hline
\end{tabular}


SCT question assessed separately

\begin{tabular}{|c|c|c|c|c|c|c|}
\hline \multirow[t]{2}{*}{ Questions } & \multicolumn{2}{|c|}{ Pre-test } & \multicolumn{2}{|c|}{ Post-test } & \multirow[t]{2}{*}{ P-value } & \multirow[t]{2}{*}{ Content } \\
\hline & $\begin{array}{l}\text { Min- } \\
\text { Max }\end{array}$ & $\begin{array}{l}\text { Median } \\
\text { (IQR) }\end{array}$ & $\begin{array}{l}\text { Min- } \\
\text { Max }\end{array}$ & $\begin{array}{l}\text { Median } \\
\text { (IQR) }\end{array}$ & & \\
\hline Q1a & $0-0.6$ & $\begin{array}{l}0.2(0.1- \\
0.6)\end{array}$ & $0-0.6$ & $\begin{array}{l}0.2(0.1- \\
0.2)\end{array}$ & $0.057^{\rrbracket}$ & \multirow[t]{3}{*}{ Confidentiality (Hep B) } \\
\hline Q1b & $0-0.5$ & $\begin{array}{l}0.2(0.2- \\
0.2)\end{array}$ & $0-0.5$ & $\begin{array}{l}0.2(0.2- \\
0.5)\end{array}$ & $0.000^{\star \star \bigotimes}$ & \\
\hline Q1c & $0-0.7$ & $\begin{array}{l}0.7(0.3- \\
0.7)\end{array}$ & $0-0.7$ & $\begin{array}{l}0.7(0.3- \\
0.7)\end{array}$ & $0.761^{\rrbracket}$ & \\
\hline Q2a & $\begin{array}{l}0.1- \\
0.4\end{array}$ & $\begin{array}{l}0.2(0.1- \\
0.4)\end{array}$ & $\begin{array}{l}0.1- \\
0.4\end{array}$ & $\begin{array}{l}0.2(0.1- \\
0.2)\end{array}$ & $0.214^{\rrbracket}$ & \multirow[t]{3}{*}{ Consent (Jehovah's witness) } \\
\hline Q2b & $0-0.4$ & $\begin{array}{l}0.2(0- \\
0.3)\end{array}$ & $0-0.4$ & $\begin{array}{l}0.3(0.1- \\
0.3)\end{array}$ & $0.340^{\rrbracket}$ & \\
\hline Q2c & $\begin{array}{l}0.1- \\
0.4\end{array}$ & $\begin{array}{l}0.3(0.1- \\
0.4)\end{array}$ & $\begin{array}{l}0.1- \\
0.4\end{array}$ & $\begin{array}{l}0.3(0.1- \\
0.4)\end{array}$ & $0.694^{\rrbracket}$ & \\
\hline Q3a & $0-0.5$ & $\begin{array}{l}0.3(0- \\
0.4)\end{array}$ & $0-0.5$ & $\begin{array}{l}0.2(0- \\
0.3)\end{array}$ & $0.008^{\text {极 }}$ & \multirow[t]{3}{*}{ Confidentiality (HIV husband) } \\
\hline Q3b & $0-0.5$ & $\begin{array}{l}0.5(0.4- \\
0.5)\end{array}$ & $0-0.5$ & $\begin{array}{l}0.5(0.4- \\
0.5)\end{array}$ & $0.100^{\rrbracket}$ & \\
\hline Q3c & $0-0.6$ & $\begin{array}{l}0.2(0- \\
0.6)\end{array}$ & $0-0.6$ & $\begin{array}{l}0.2(0.2- \\
0.6)\end{array}$ & $0.893^{\rrbracket}$ & \\
\hline Q4a & $0-0.4$ & $\begin{array}{l}0.3(0.2- \\
0.4)\end{array}$ & $0-0.4$ & $\begin{array}{l}0.3(0.2- \\
0.4)\end{array}$ & $0.392^{\bigotimes}$ & \multirow[t]{3}{*}{ Consent (surgery in Old female) } \\
\hline Q4b & $0-0.5$ & $\begin{array}{l}0.2(0- \\
0.2)\end{array}$ & $0-0.7$ & $\begin{array}{l}0.2(0.1- \\
0.7)\end{array}$ & $0.506^{\rrbracket}$ & \\
\hline Q4c & $0-0.7$ & $\begin{array}{l}0.2(0- \\
0.7)\end{array}$ & $0-0.5$ & $\begin{array}{l}0.2(0- \\
0.3)\end{array}$ & $0.169^{\rrbracket}$ & \\
\hline Q5a & $0-0.5$ & $\begin{array}{l}0.2(0- \\
0.3)\end{array}$ & $0-0.3$ & $\begin{array}{l}0.2(0.2- \\
0.3)\end{array}$ & $0.141^{\llbracket}$ & \multirow[t]{3}{*}{$\begin{array}{l}\text { Whistle blowing (medical } \\
\text { negligence) }\end{array}$} \\
\hline Q5b & $0-0.3$ & $\begin{array}{l}0.2(0.2- \\
0.3)\end{array}$ & $0-0.3$ & $\begin{array}{l}0.2(0.2- \\
0.3)\end{array}$ & $0.179^{\rrbracket}$ & \\
\hline Q5c & $0-1$ & $1(0-1)$ & $0-1$ & $1(0-1)$ & $0.724^{\Downarrow}$ & \\
\hline Q6a & $0-0.6$ & $\begin{array}{l}0.1(0- \\
0.4)\end{array}$ & $0-0.4$ & $\begin{array}{l}0.3(0.1- \\
0.3)\end{array}$ & $0.000^{\star \star \rrbracket}$ & \multirow[t]{2}{*}{$\begin{array}{l}\text { Breaking bad news (old female } \\
\text { with mets) }\end{array}$} \\
\hline Q6b & $0-0.4$ & $\begin{array}{l}0.3(0.1- \\
0.3)\end{array}$ & $0-0.4$ & $\begin{array}{l}0.3(0.1- \\
0.3)\end{array}$ & $0.697^{\square}$ & \\
\hline
\end{tabular}




\begin{tabular}{|c|c|c|c|c|c|c|}
\hline \multirow[t]{2}{*}{ Questions } & \multicolumn{2}{|c|}{ Pre-test } & \multicolumn{2}{|c|}{ Post-test } & \multirow[t]{2}{*}{ P-value } & \multirow[t]{2}{*}{ Content } \\
\hline & $\begin{array}{l}\text { Min- } \\
\text { Max }\end{array}$ & $\begin{array}{l}\text { Median } \\
\text { (IQR) }\end{array}$ & $\begin{array}{l}\text { Min- } \\
\text { Max }\end{array}$ & $\begin{array}{l}\text { Median } \\
\text { (IQR) }\end{array}$ & & \\
\hline Q6c & $\begin{array}{l}0.1- \\
0.5\end{array}$ & $\begin{array}{l}0.1(0.1- \\
0.5)\end{array}$ & $\begin{array}{l}0.1- \\
0.5\end{array}$ & $\begin{array}{l}0.1(0.1- \\
0.5)\end{array}$ & $0.030^{\star \rrbracket}$ & \\
\hline Q7a & $\begin{array}{l}0.1- \\
0.4\end{array}$ & $\begin{array}{l}0.1(0.1- \\
0.3)\end{array}$ & $\begin{array}{l}0.1- \\
0.4\end{array}$ & $\begin{array}{l}0.1(0.1- \\
0.3)\end{array}$ & $0.034^{\text {*㕅 }}$ & \multirow[t]{3}{*}{$\begin{array}{l}\text { Beginning of life care (termination } \\
\text { of pregnancy) }\end{array}$} \\
\hline Q7b & $\begin{array}{l}0.1- \\
0.4\end{array}$ & $\begin{array}{l}0.2(0.1- \\
0.3)\end{array}$ & $\begin{array}{l}0.1- \\
0.4\end{array}$ & $\begin{array}{l}0.2(0.1- \\
0.2)\end{array}$ & $0.016^{\text {*㕅 }}$ & \\
\hline Q7c & $0-0.4$ & - & $0-0.4$ & - & $0.334^{\rrbracket}$ & \\
\hline Q8a & $0-0.4$ & $\begin{array}{l}0.2(0- \\
0.3)\end{array}$ & $0-0.4$ & $\begin{array}{l}0.2(0- \\
0.3)\end{array}$ & $0.288^{\rrbracket}$ & \multirow[t]{3}{*}{$\begin{array}{l}\text { Conflict of interest (Dubai trip for } \\
\text { junior doctor) }\end{array}$} \\
\hline Q8b & $0-0.4$ & $\begin{array}{l}0.1(0- \\
0.3)\end{array}$ & $0-0.4$ & $\begin{array}{l}0.2(0- \\
0.3)\end{array}$ & $0.832^{\bigotimes}$ & \\
\hline Q8c & $0-0.7$ & $\begin{array}{l}0.2(0.1- \\
0.7)\end{array}$ & $0-0.7$ & $\begin{array}{l}0.7(0.2- \\
0.7)\end{array}$ & $0.019^{\text {*区 }}$ & \\
\hline Q9a & $0-0.6$ & $\begin{array}{l}0.6(0.3- \\
0.6)\end{array}$ & $0-0.6$ & $\begin{array}{l}0.6(0.4- \\
0.6)\end{array}$ & $0.163^{\llbracket}$ & \multirow[t]{3}{*}{$\begin{array}{l}\text { Public health (immunisation of } \\
\text { one yr. old) }\end{array}$} \\
\hline Q9b & $0-0.5$ & - & $0-0.5$ & - & $0.634^{\rrbracket}$ & \\
\hline Q9c & $0-0.5$ & $\begin{array}{l}0.3(0.1- \\
0.5)\end{array}$ & $0-0.5$ & $\begin{array}{l}0.1(0.1- \\
0.3)\end{array}$ & $0.167^{\rrbracket}$ & \\
\hline Q10a & $0-0.4$ & $\begin{array}{l}0.2(0- \\
0.4)\end{array}$ & $0-0.4$ & $\begin{array}{l}0.2(0- \\
0.4)\end{array}$ & $0.198^{\rrbracket}$ & \multirow[t]{3}{*}{ Publication ethics (plagiarism) } \\
\hline Q10b & $0-0.5$ & $\begin{array}{l}0.3(0.2- \\
0.5)\end{array}$ & $0-0.5$ & $\begin{array}{l}0.3(0.2- \\
0.5)\end{array}$ & $0.847^{\rrbracket}$ & \\
\hline Q10c & $0-0.4$ & $\begin{array}{l}0.4(0.1- \\
0.4)\end{array}$ & $0-0.4$ & $\begin{array}{l}0.4(0.1- \\
0.4)\end{array}$ & $0.008^{\text {*㕅 }}$ & \\
\hline Q11a & $0-0.3$ & $\begin{array}{l}0.2(0- \\
0.3)\end{array}$ & $0-0.3$ & $\begin{array}{l}0.2(0- \\
0.3)\end{array}$ & $0.382^{\rrbracket}$ & \multirow[t]{3}{*}{$\begin{array}{l}\text { Breaking bad news (friend with } \\
\text { carcinoma) }\end{array}$} \\
\hline Q11b & $\begin{array}{l}0.1- \\
0.3\end{array}$ & $\begin{array}{l}0.2(0.1- \\
0.3)\end{array}$ & $\begin{array}{l}0.1- \\
0.3\end{array}$ & $\begin{array}{l}0.2(0.1- \\
0.3)\end{array}$ & $0.684^{\rrbracket}$ & \\
\hline Q11c & $0-0.4$ & $\begin{array}{l}0.3(0.1- \\
0.4)\end{array}$ & $0-0.4$ & $\begin{array}{l}0.3(0.1- \\
0.4)\end{array}$ & $1.000^{\rrbracket}$ & \\
\hline Q12a & $0-0.4$ & $\begin{array}{l}0.3(0- \\
0.4)\end{array}$ & $0-0.4$ & $0(0-0.3)$ & $0.006^{\text {极 }}$ & \multirow[t]{2}{*}{$\begin{array}{l}\text { Care of the vulnerable ( } 3 \text { year. old } \\
\text { with meningitis) }\end{array}$} \\
\hline Q12b & $0-0.6$ & $\begin{array}{l}0.3(0- \\
0.6)\end{array}$ & $0-0.6$ & $\begin{array}{l}0.3(0- \\
0.6)\end{array}$ & $0.009^{\star \rrbracket}$ & \\
\hline
\end{tabular}




\begin{tabular}{|c|c|c|c|c|c|c|}
\hline \multirow[t]{2}{*}{ Questions } & \multicolumn{2}{|c|}{ Pre-test } & \multicolumn{2}{|c|}{ Post-test } & \multirow[t]{2}{*}{ P-value } & \multirow[t]{2}{*}{ Content } \\
\hline & $\begin{array}{l}\text { Min- } \\
\text { Max }\end{array}$ & $\begin{array}{l}\text { Median } \\
\text { (IQR) }\end{array}$ & $\begin{array}{l}\text { Min- } \\
\text { Max }\end{array}$ & $\begin{array}{l}\text { Median } \\
\text { (IQR) }\end{array}$ & & \\
\hline Q12C & $0-0.4$ & $\begin{array}{l}0.3(0.2- \\
0.4)\end{array}$ & $0-0.4$ & $\begin{array}{l}0.3(0.2- \\
0.4)\end{array}$ & $0.882^{\bigotimes}$ & \\
\hline Q13a & $\begin{array}{l}0.1- \\
0.3\end{array}$ & $\begin{array}{l}0.2(0.2- \\
0.2)\end{array}$ & $\begin{array}{l}0.1- \\
0.3\end{array}$ & $\begin{array}{l}0.2(0.2- \\
0.2)\end{array}$ & $0.266^{\rrbracket}$ & \multirow[t]{3}{*}{$\begin{array}{l}\text { Breaking bad news (man with } \\
\text { mets) }\end{array}$} \\
\hline Q13b & $0-0.4$ & $\begin{array}{l}0.3(0.1- \\
0.4)\end{array}$ & $0-0.4$ & $\begin{array}{l}0.3(0.1- \\
0.4)\end{array}$ & $0.554^{\rrbracket}$ & \\
\hline Q13c & $0-0.7$ & $\begin{array}{l}0.1(0.1- \\
0.1)\end{array}$ & $0-0.7$ & $\begin{array}{l}0.1(0.1- \\
0.7)\end{array}$ & $0.190^{\rrbracket}$ & \\
\hline Q14a & $0-0.8$ & $\begin{array}{l}0.2(0- \\
0.8)\end{array}$ & $0-0.8$ & $\begin{array}{l}0.2(0- \\
0.8)\end{array}$ & $0.011^{\text {*㕅 }}$ & \multirow[t]{3}{*}{$\begin{array}{l}\text { Research ethics (consent from } \\
\text { minors) }\end{array}$} \\
\hline Q14b & $0-0.6$ & $\begin{array}{l}0.1(0- \\
0.3)\end{array}$ & $0-0.6$ & $\begin{array}{l}0.3(0- \\
0.6)\end{array}$ & $0.016^{\text {极 }}$ & \\
\hline Q14c & $0-0.4$ & - & $0-0.4$ & - & $0.620^{\square}$ & \\
\hline Q15a & $0-0.7$ & $\begin{array}{l}0.2(0- \\
0.7)\end{array}$ & $0-0.7$ & $\begin{array}{l}0.1(0- \\
0.7)\end{array}$ & $0.480^{\rrbracket}$ & \multirow[t]{3}{*}{ Conflict of interest (friend's lab) } \\
\hline Q15b & $0-0.5$ & $\begin{array}{l}0.3(0.2- \\
0.5)\end{array}$ & $0-0.5$ & $\begin{array}{l}0.3(0.2- \\
0.4)\end{array}$ & $0.190^{\rrbracket}$ & \\
\hline Q15c & $0-0.6$ & $\begin{array}{l}0.4(0- \\
0.6)\end{array}$ & $0-0.6$ & $\begin{array}{l}0.4(0.4- \\
0.4)\end{array}$ & $0.421^{\rrbracket}$ & \\
\hline Q16a & $0-0.5$ & $\begin{array}{l}0.3(0- \\
0.5)\end{array}$ & $0-0.5$ & $\begin{array}{l}0.3(0.2- \\
0.5)\end{array}$ & $0.068^{\rrbracket}$ & \multirow[t]{3}{*}{ Confidentiality (when to breach?) } \\
\hline Q16b & $0-0.8$ & $\begin{array}{l}0.8(0.2- \\
0.8)\end{array}$ & $0-0.8$ & $\begin{array}{l}0.8(0.2- \\
0.8)\end{array}$ & $0.080^{\rrbracket}$ & \\
\hline Q16c & $0-0.5$ & $\begin{array}{l}0.1(0.1- \\
0.5)\end{array}$ & $0-0.5$ & $\begin{array}{l}0.3(0.1- \\
0.5)\end{array}$ & $0.010^{\star \rrbracket}$ & \\
\hline Q17a & $0-0.4$ & $\begin{array}{l}0.2(0.1- \\
0.3)\end{array}$ & $0-0.4$ & $\begin{array}{l}0.3(0.1- \\
0.3)\end{array}$ & $0.048^{\text {*囚 }}$ & \multirow[t]{3}{*}{$\begin{array}{l}\text { Truth telling (school sick-leave } \\
\text { certificate) }\end{array}$} \\
\hline Q17b & $0-0.9$ & $\begin{array}{l}0.1(0- \\
0.9)\end{array}$ & $0-0.9$ & $\begin{array}{l}0.1(0- \\
0.9)\end{array}$ & $0.582^{\rrbracket}$ & \\
\hline Q17c & $0-0.7$ & $\begin{array}{l}0.1(0.1- \\
0.7)\end{array}$ & $0-0.7$ & $\begin{array}{l}0.1(0.1- \\
0.7)\end{array}$ & $0.941^{\rrbracket}$ & \\
\hline Q18a & $0-0.5$ & $\begin{array}{l}0.2(0.2- \\
0.3)\end{array}$ & $0-0.5$ & $\begin{array}{l}0.3(0.2- \\
0.5)\end{array}$ & $0.385^{\rrbracket}$ & \multirow[t]{2}{*}{ Medical Error (reporting) } \\
\hline Q18b & $0-0.3$ & $\begin{array}{l}0.2(0.2- \\
0.3)\end{array}$ & $0-0.3$ & $\begin{array}{l}0.2(0.2- \\
0.3)\end{array}$ & $0.427^{\rrbracket}$ & \\
\hline
\end{tabular}




\begin{tabular}{|c|c|c|c|c|c|c|}
\hline \multirow[t]{2}{*}{ Questions } & \multicolumn{2}{|c|}{ Pre-test } & \multicolumn{2}{|c|}{ Post-test } & \multirow[t]{2}{*}{ P-value } & \multirow[t]{2}{*}{ Content } \\
\hline & $\begin{array}{l}\text { Min- } \\
\text { Max }\end{array}$ & $\begin{array}{l}\text { Median } \\
\text { (IQR) }\end{array}$ & $\begin{array}{l}\text { Min- } \\
\text { Max }\end{array}$ & $\begin{array}{l}\text { Median } \\
\text { (IQR) }\end{array}$ & & \\
\hline Q18c & $0-0.4$ & $\begin{array}{l}0.3(0.1- \\
0.4)\end{array}$ & $0-0.4$ & $\begin{array}{l}0.3(0.1- \\
0.4)\end{array}$ & $0.897^{\rrbracket}$ & \\
\hline Q19a & $\begin{array}{l}0.1- \\
0.3\end{array}$ & - & $\begin{array}{l}0.1- \\
0.3\end{array}$ & - & $0.219^{\rrbracket}$ & Publication ethics (authorship) \\
\hline Q19b & $0-0.6$ & $\begin{array}{l}0.4(0- \\
0.6)\end{array}$ & $0-0.6$ & $\begin{array}{l}0.4(0.4- \\
0.6)\end{array}$ & $0.268^{\rrbracket}$ & \\
\hline Q19c & $0-0.4$ & $\begin{array}{l}0.2(0.1- \\
0.3)\end{array}$ & $0-0.4$ & $\begin{array}{l}0.2(0.1- \\
0.3)\end{array}$ & $0.759^{\rrbracket}$ & \\
\hline Q20a & $0-0.4$ & $\begin{array}{l}0.4(0.1- \\
0.4)\end{array}$ & $0-0.4$ & $\begin{array}{l}0.1(0.1- \\
0.4)\end{array}$ & $0.390^{\rrbracket}$ & End of life care (pulling the plug) \\
\hline Q20b & $0-0.5$ & - & $0-0.5$ & - & $0.061^{\square}$ & \\
\hline Q20c & $0-0.6$ & $\begin{array}{l}0.4(0- \\
0.6)\end{array}$ & $0-0.6$ & $0(0-0.4)$ & $0.384^{\rrbracket}$ & \\
\hline
\end{tabular}


Table 5

KFQ items assessed separately

\begin{tabular}{|c|c|c|c|c|c|}
\hline & & Pre test & & & $P$ value \\
\hline & & 0 & 1 & Total & \\
\hline & & $N(\%)$ & $N(\%)$ & $N(\%)$ & \\
\hline 21A Truth & elling/ & espect for & uman dign & y/ autonom & \\
\hline Post test & 0 & 10(11.1) & $5(5.6)$ & 15(16.7) & $0.000^{\star \star \bigotimes}$ \\
\hline & 1 & $28(31.1)$ & $47(52.2)$ & 75(83.3) & \\
\hline & Total & $38(42.2)$ & $52(57.8)$ & $90(100)$ & \\
\hline 21B Cons & & & & & \\
\hline Post test & 0 & $19(21.1)$ & $10(11.1)$ & $29(32.2)$ & $0.050^{\square}$ \\
\hline & 1 & $22(24.4)$ & $39(43.3)$ & $61(67.8)$ & \\
\hline & Total & $41(45.6)$ & $49(54.4)$ & $90(100)$ & \\
\hline 22A Doctc & -patient & elationshi & & & \\
\hline Post test & 0 & $34(37.8)$ & $11(12.2)$ & $45(50)$ & $0.265^{\square}$ \\
\hline & 1 & $18(20)$ & $27(30)$ & $45(50)$ & \\
\hline & Total & $52(57.8)$ & $38(42.2)$ & $90(100)$ & \\
\hline 22B Truth & elling/ & espect for & uman dign & y/ autonom & \\
\hline Post test & 0 & $46(51.1)$ & $13(14.4)$ & $59(65.6)$ & $0.711^{\square}$ \\
\hline & 1 & $16(17.8)$ & 15(16.7) & $31(34.4)$ & \\
\hline & Total & $62(68.9)$ & $28(31.1)$ & $90(100)$ & \\
\hline 23A Cons & & & & & \\
\hline Post test & 0 & 11(12.2) & $6(6.7)$ & 17(18.9) & $0.000^{\star \star \bigotimes}$ \\
\hline & 1 & $48(53.3)$ & $25(27.8)$ & 73(81.1) & \\
\hline & Total & $59(65.6)$ & $31(34.4)$ & $90(100)$ & \\
\hline 23B Respe & t for ho & nan dignit) & autonom & & \\
\hline Post test & 0 & $27(30)$ & 12(13.3) & $39(43.3)$ & $0.005^{\star \star \rrbracket}$ \\
\hline & 1 & $31(34.4)$ & $20(22.2)$ & $51(56.7)$ & \\
\hline & Total & $58(64.4)$ & $32(35.6)$ & $90(100)$ & \\
\hline
\end{tabular}




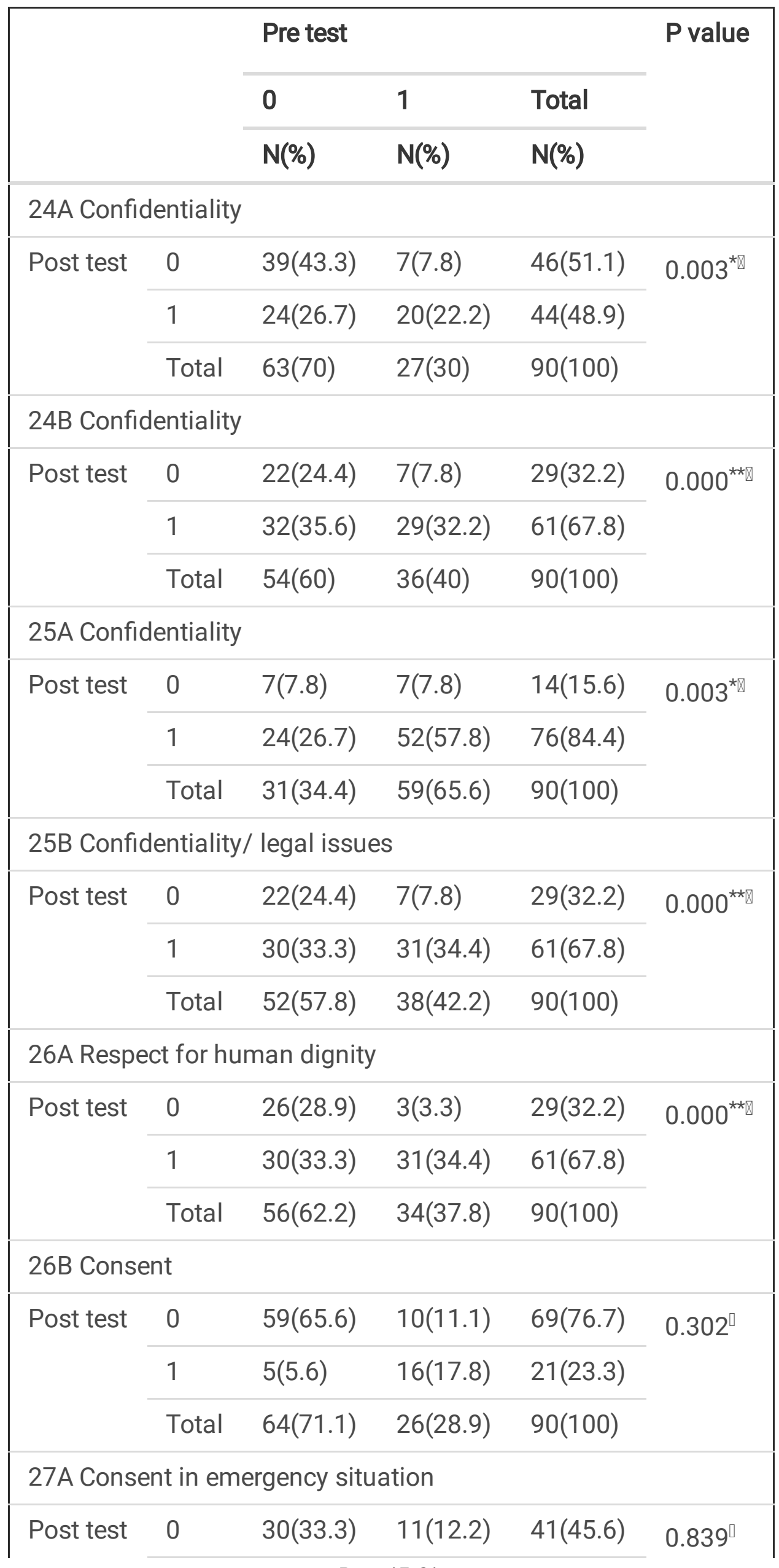




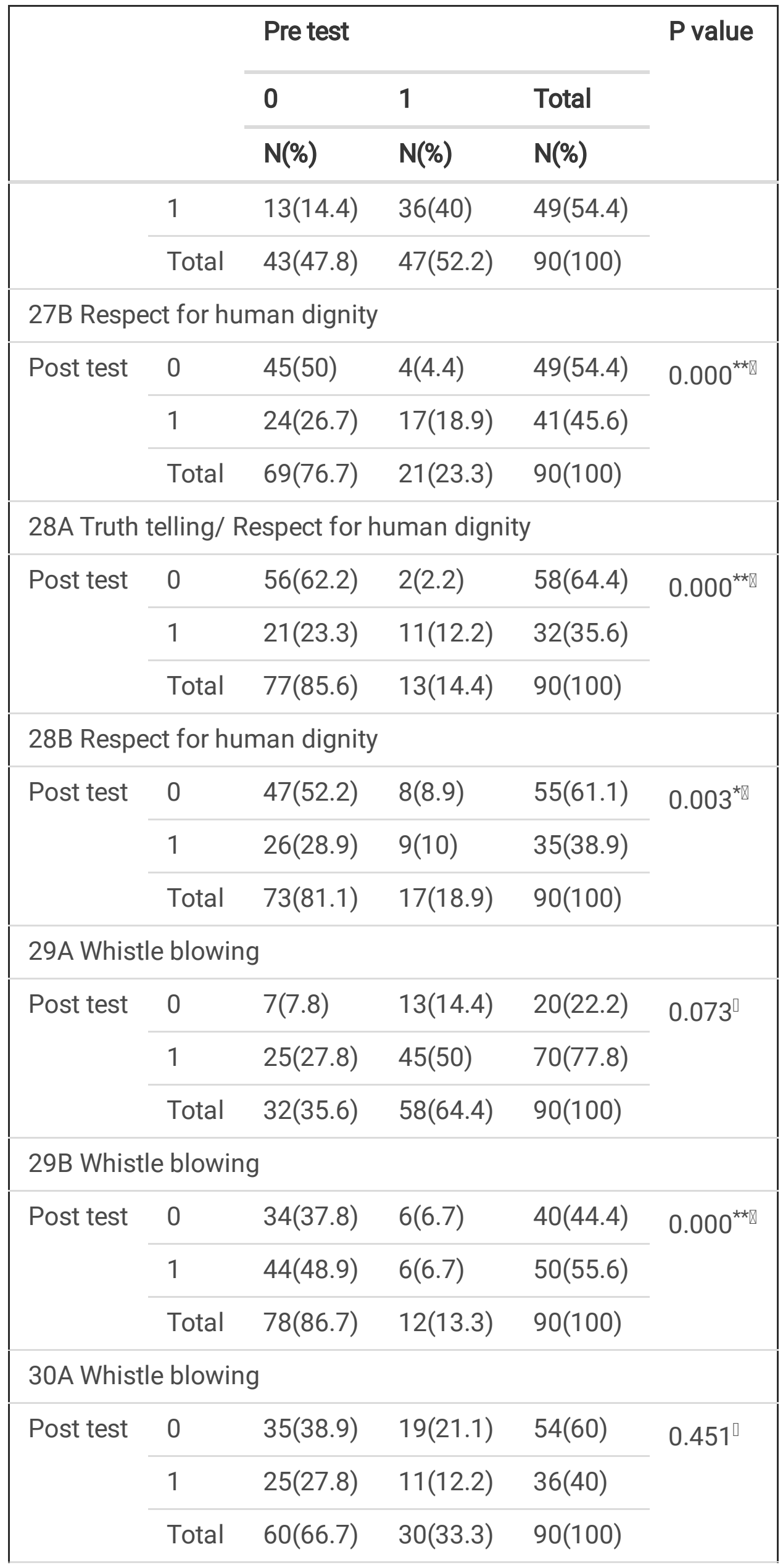




\begin{tabular}{|c|c|c|c|c|c|}
\hline & & \multicolumn{3}{|l|}{ Pre test } & \multirow[t]{3}{*}{$P$ value } \\
\hline & & 0 & 1 & Total & \\
\hline & & $N(\%)$ & $N(\%)$ & $N(\%)$ & \\
\hline \multicolumn{6}{|c|}{ 30B Ttruth telling } \\
\hline \multirow[t]{3}{*}{ Post test } & 0 & $53(58.9)$ & $9(10)$ & $62(68.9)$ & \multirow[t]{3}{*}{$0.061^{\square}$} \\
\hline & 1 & $20(22.2)$ & $8(8.9)$ & 28(31.1) & \\
\hline & Total & 73(81.1) & 17(18.9) & $90(100)$ & \\
\hline
\end{tabular}

The CFQ showed high reliability of 0.95 using Cronbach's Alpha, with all components contributing equally to the questionnaire's reliability. The feedback showed that most participants $(97 \%)$ considered the course to be of value. Ninety-four per cent (94\%) thought it improved their critical thinking and that it was useful. Eighty-nine per cent (89\%) found the WBEL to be a valuable learning strategy during the course. Students appreciated the use of diverse methods employed for the facilitation of learning. Study participants highly appreciated case discussions using case vignettes (91\%) and video clips (87\%) for student engagement and active learning. A significant majority, $84 \%$ and $78 \%$, considered classroom quizzes and reflective writing as worthwhile for learning during the course.

\section{Discussion}

The purpose of this study was to assess the impact of WBEL, a novel educational strategy, on students' ethics-relatedknowledge and course engagement, in the context of the region of the Middle East and South Asia. The countries in the Middle East and South Asian regions share commonalities that set them apart from developed western cultures in relation to this component of medical programs. The differences are in terms of societal norms, politics, legislation, beliefs, traditions and lifestyles [45]. It is important to note that these regions also have a substantial global impact on medical education as they contain $60 \%$ of the medical institutes producing medical graduates [46]. These graduates provide healthcare services to a large local population within the region and form a significant portion of international medical graduates (IMGs) serving in the developed western World [47]. For example, an estimated 45 per cent of the IMGs in the USA originate from the Middle East and South Asian regions [48].

As noted previously, several countries in the Middle East and South Asian regions face resource constraints and gaps that impact undergraduate medical ethics education. Among these constraints, the lack of culturally relevant texts is significant, as contemporary medical ethics has not developed indigenously and instead is considered an imported western concept $[12,13]$. In support of this view, educators in Saudi Arabia [4] and Pakistan [49] argue that the importation of a secular western 
curriculum of ethics is not in accord with the religious teachings in the region. They emphasise that ethics education requires an inclusive, more reflective, and socially relevant approach to which the students can relate. Several other medical ethics educators in the region also opine that the Anglo-European ethical traditions that shape contemporary medical ethics education are not sufficient to encompass the indigenous values and beliefs of students in these regions [4, 14, 49-51]. Other constraints that have been identified as hampering the integration of medical ethics into the curriculum include a lack of competent, dedicated medical ethics teachers and often indifferent institutional policies [2, 6-9].

This study shows that the WBEL strategy is an enabler for the delivery of ethics education, and helps overcome the above resource constraints by creating appropriate content and methods through a robust consultative process [17]. The inclusion of focussed reading material and social interaction with peers and faculty in the WBEL model explicitly compensates for the lack of learning resources relevant to the regions $[52,53]$. The strategy is further strengthened by the inclusion of reflective writing and feedback towards the end of the learning process. The reflection and feedback process also supports the students in assessing their learning by articulating how they have approached the given task using their previous knowledge and understanding of the new information [54]. The iterative refinement of this strategy with input from external experts, local faculty, and students (Shamim et al., 2021) is likely to enhance the strategy's effectiveness.

One of the aims of this study was to evaluate students' engagement in terms of satisfaction and enjoyability with this novel teaching strategy. Students' satisfaction and enjoyability have shown to be related to the diversity of the methods used in their learning process [55]. In addition to offering diversity to the learning process, the use of various teaching methods effectively covers different aspects of ethics education, like concepts, analysis, decision-making, that require distinct teaching methods for effective learning [56]. The feedback from students enrolled in this study showed an overwhelmingly positive response towards the diverse educational methods, like small group discussions, role-plays and video demonstrations, used in the ethics course.

Karagiorgi and Symeou (2005) suggest that students take ownership of problems or tasks and provide authentic, sound solutions when these are closer to their reality. For example, students find narration of problems that originate from their facilitators' or their own experiences as most stimulating and authentic $[57,58]$. Therefore, in accordance with the CREEM framework, each session in the medical ethics course in this study started the learning process with a socio-culturally relevant experience based within an environment familiar to the students. The participants of this study acknowledged the value of this ownership and relevance. In other words, the ethics course provided the students with an authentic experience to construct their further learning.

In this study, the pre-and post-test, using KFQ and SCT items, systematically assessed the impact of the intervention. KFQ is focused on challenging aspects of clinical problems where learners are more likely to make errors [59]. Similarly, based on script theory in cognitive psychology (Charlin et al., 2000), SCT has shown to assess students' organisation of ethical knowledge while making decisions [31]. The 
combination of the KFQ with SCT as pre-post-test measures provided a robust method to evaluate the impact of WBEL on developing ethical reasoning skills (abilities to identify ethical dilemmas and make decisions in a situation of uncertainty).

Although the course faculty belonged to diverse disciplines, without a primary qualification in ethics, the knowledge and guidance they received through the Workbook empowered them to deliver the course content effectively. The resource material in the Workbook informed the faculty about the scope and depth of content needed while sharing their experiences during the discourse. A shortage of medical teachers who are specifically trained to teach medical ethics is not a new [60] or isolated [61] phenomenon and has previously been identified as a resource constraint in medical ethics education in various contexts $[8,62,63]$. Students' feedback regarding the facilitators' performance during the course suggests that the provision of the Workbook and guidance for facilitators helped them overcome this constraint to some extent. Students' positive feedback about the overall ethics course and appreciation of different methods used was also seen as a source of encouragement to the faculty involved in delivering the ethics course in this study.

The findings of this study, thus, make some significant contributions to the literature. Firstly, the findings illustrate the practical relevance of the CREEM framework and WBEL strategy for the delivery of medical ethics education in the studied context. Secondly, the findings are likely to apply to othercontexts with similarities to the study's regions. Last but not least, the paper contributes to the existing literature by providing initial evidence on the use of an innovative educational strategy for contextually relevant ethics education, thereby creating avenues for further contextually relevant research in medical ethics education.

\section{Limitations}

The study found significant improvements in overall pre- to post- measures; however, the differences were more remarkable for the KFQs, while their performance in SCTs was weak. One reason for this discrepancy may be that the students at both sites were unfamiliar with SCTs as a testing method. It can be inferred that, as for any assessment tool, although SCTs have evidence of validity, they may not perform well with test-takers if they are not familiar with the tool [31, 64].

This study tested the WBEL in the context of only two institutes. The study participants were predominantly females in the Pakistan cohort and all males in Saudi Arabia. This gender imbalance was beyond the researchers' control. Nevertheless, the similarity of social contexts in these countries and nearly equal gender representation in the combined sample of participants may support the generalizability of results in countries with similar social context. However, these are still considered as limitations of this study. The impact of the intervention on medical ethics education generally will need further exploration.

\section{Conclusion}


The study identifies WBEL as a promising way forward in delivering medical ethics education by demonstrating notable improvement in participants' knowledge and ethical decision-making skills. The Workbook design and its use within the WBEL strategy will be particularly beneficial for teaching ethics in regions where contextually relevant local reading material on healthcare ethics and specifically trained ethics educators are not readily available for medical students. The use and evaluation of the WBEL in other similar regions would generate further evidence of this strategy's wider applicability.

\section{List Of Abbreviations (In Alphabetical Order)}

AT - Adrienne Torda

CB - Chinthaka Balasooriya

CFQ - Course Feedback Questionnaire

CREEM - Contextually Relevant Ethics Education Model

FQ 2 - Feedback Questionnaire 2

IMG - International Medical Graduates

JSMU - Jinnah Sindh Medical University

KAU - King Abdulaziz University

KFQ - Key Feature Questions

LB - Lubna Baig

MSLQ - Motivated Strategies for Learning Questionnaire

MSS - Muhammad Shahid Shamim

SCT - Script Concordance Test

UNSW - University of New South Wales

WBEL - Workbook based ethics learning

\section{Declarations}

\section{Ethics approval}

The ethics approval for this study was acquired from the ethics review committee of King Abdulaziz University (reference no. 393-15), Jinnah Sindh Medical University (reference no. 2016-30), where the 
study was conducted, and the UNSW (approval no. HC15640) Australia, where the primary author is enrolled as a PhD scholar.

\section{Consent for participation and publication}

The potential participants were informed about the researchers and the study in detail. The Participant Information Statement and Consent Form prescribed by UNSW was used to seek informed consent for the study and publication from students and faculty participating in the study. All the data were anonymized prior to analysis.

The authors further confirm that all methods were performed in accordance with the relevant guidelines and regulations of UNSW.

\section{Availability of data and material}

All the data from this study is submitted to the UNSW compactus as per the University policy for safeguarding and future retrieval. The data that support the findings of this study are available from UNSW but restrictions apply to the availability of these data as they are not publicly available. Data are however available from the authors, MSS and CB, upon reasonable request and with permission of UNSW.

\section{Competing interests}

The primary author was a PhD scholar at the University of New South Wales (UNSW), Sydney, Australia. This study is a part of the PhD thesis of the primary author.

There is no conflict of interest.

\section{Funding}

The study or any part of it is not funded by any source. The primary author was a fee-paying PhD candidate at UNSW and is personally baring all expenses related to the research and publication.

\section{Authors' contribution}

All authors have significantly contributed to the study design, analysis and development of the manuscript for publication. The analysis was reviewed and improved by other authors. The first draft of the manuscript was prepared by MSS. All the other authors reviewed, revised and provided their input in improving the manuscript.

MSS Shamim was involved in conception of research idea, design of the work, the acquisition, analysis and interpretation of data. He prepared the first draft of the manuscript. As the corresponding author, he ensured that all listed authors have approved the manuscript before submission, including the names and order of authors, and that all authors receive the submission and all substantive correspondence with 
editors, as well as the full reviews, verifying that all data and figures comply with the transparency and reproducibility standards.

LB contributed to developing the design of work and analyses and interpretation of the data. She substantially participated in reviewing the manuscript.

AT was involved in the development of the conceived idea and design of the work. she substantially participated in drafting and reviewing the manuscript.

CB contributed significantly to the development of the conceived idea and design of the work. He supervised the overall research process, analysis of data and substantially participated in reviewing and revising the manuscript.

All the authors have approved the submitted versions. The have agreed both to be personally accountable for the author's own contributions and to ensure that questions related to the accuracy or integrity of any part of the work are appropriately investigated and resolved.

\section{Acknowledgements}

Faisal Ghani for organising and Aamir Jaffary, Abeer, Quaratul Ain for co-facilitating the ethics course at JSMU.

Muhammad Farooq for organising and Nadeem Zubairi, Muhammad Imran and Marwan Bakkerman for co-facilitating the ethics course at KAU.

Sundus Iftikhar, Nida Ghouri and Mukhtiar Baig for helping with statistical analysis.

\section{References}

1. Campbell, A.V., J. Chin, and T.-C. Voo, How can we know that ethics education produces ethical doctors? Medical teacher, 2007. 29(5): p. 431-436.

2. de la Garza, S., et al., Teaching medical ethics in graduate and undergraduate medical education: a systematic review of effectiveness. Academic Psychiatry, 2017. 41(4): p. 520-525.

3. Passi, V., et al., Developing medical professionalism in future doctors: a systematic review. International Journal of Medical Education, 2010. 1: p. 19.

4. Alkabba, A.F., et al., Teaching and evaluation methods of medical ethics in the Saudi public medical colleges: cross-sectional questionnaire study. BMC Med Educ, 2013. 13: p. 122.

5. Ekmekci, P.E., Medical ethics education in Turkey; state of play and challenges. International online journal of education \& teaching, 2016. 3(1): p. 54.

6. Hoffmann, W.A. and N. Nortjé, Curriculum Guidelines for Teaching Healthcare Ethics at Undergraduate Level, in African Perspectives on Ethics for Healthcare Professionals. 2018, Springer. p. 251-266. 
7. Kavas, M.V., et al., The state of ethics education at medical schools in Turkey: taking stock and looking forward. BMC Medical Education, 2020. 20: p. 1-15.

8. Wong, M.K., et al., Teaching Ethics in Medical Schools: A Systematic Review from 2000 to 2018. Journal of Medical Education, 2020. 18(4).

9. Merrick, A., et al., Introducing the medical ethics bowl. Cambridge Q. Healthcare Ethics, 2016. 25: p. 141.

10. Khizar, B. and M. Iqbal, Mock ethics consultations for teaching ethics in Pakistan. Medical education, 2009. 43(5): p. 477-477.

11. Stikkers, K.W., John Dewey: education as ethics, ethics as education. Pedagogical Culture, 2017. 2(1): p. 39-49.

12. Mohammadifar, S. and L. Afshar, Comparative Study of Medical Ethics Curriculum in General MedicineCourse in 10Selected Universities in the World. Journal of Medical Education, 2019. 18(1).

13. Jha, V., et al., Medical professionalism across cultures: A challenge for medicine and medical education. Medical teacher, 2015. 37(1): p. 74-80.

14. Shamim, M., et al., Culture and ethics in medical education: The Asian perspective. JPMA. The Journal of the Pakistan Medical Association, 2018. 68(3): p. 444-446.

15. Pratt, B., et al., Perspectives from South and East Asia on clinical and research ethics: a literature review. J Empir Res Hum Res Ethics, 2014. 9(2): p. 52-67.

16. Shamim, M.S., et al., Systematic Development and Refinement of a Contextually Relevant Strategy for Undergraduate Medical Ethics Education: a qualitative study. 2020.

17. Shamim, M.S., et al., Systematic development and refinement of a contextually relevant strategy for undergraduate medical ethics education: a qualitative study. BMC Medical Education, 2021. 21(1): p. $1-12$.

18. Shamim, M.S., A contextually relevant ethics education model. International Journal of Ethics Education, 2020: p. 1-4.

19. Faul, F., et al., $G *$ Power 3: A flexible statistical power analysis program for the social, behavioral, and biomedical sciences. Behavior research methods, 2007. 39(2): p. 175-191.

20. Cohen, J., Statistical power analysis. Current directions in psychological science, 1992. 1(3): p. 98101.

21. Dumas, J.-P., J.-G. Blais, and B. Charlin, Script concordance test: can it be used to assess clinical reasoning of physiotherapy student? Physiotherapy, 2015. 101: p. e332-e333.

22. Chang, T.P., et al., Script concordance testing: assessing residents' clinical decision-making skills for infant lumbar punctures. Academic Medicine, 2014. 89(1): p. 128-135.

23. Wan, M.S.H., E. Tor, and J.N. Hudson, Construct validity of script concordance testing: progression of scores from novices to experienced clinicians. International journal of medical education, 2019. 10 : p. 174. 
24. Jost, M., et al., Effects of additional team-based learning on students' clinical reasoning skills: a pilot study. BMC research notes, 2017. 10(1): p. 1-7.

25. Ferreira-Padilla, G., et al., Ethics competences in the undergraduate medical education curriculum: the Spanish experience. Croatian medical journal, 2016. 57(5): p. 493-503.

26. Paas, F., A. Renkl, and J. Sweller, Cognitive load theory and instructional design: Recent developments. Educational psychologist, 2003. 38(1): p. 1-4.

27. Sweller, J., J.J. van Merriënboer, and F. Paas, Cognitive architecture and instructional design: 20 years later. Educational Psychology Review, 2019: p. 1-32.

28. Ayres, P. and F. Paas, Cognitive load theory: New directions and challenges. Applied Cognitive Psychology, 2012. 26(6): p. 827-832.

29. Biggs, J., Enhancing teaching through constructive alignment. Higher education, 1996. 32(3): p. 347-364.

30. Raskin, J.D. and A.E. Debany, The inescapability of ethics and the impossibility of "anything goes": A constructivist model of ethical meaning making. Journal of Constructivist Psychology, 2018. 31(4): p. 343-360.

31. Pau, A., et al., Development and Validation of a Script Concordance Test (SCT) to Evaluate Ethical Reasoning Ability Among First and Fifth Year Students in a Medical School. Journal of Academic Ethics, 2019. 17(2): p. 193-204.

32. Charlin, B., et al., The Script Concordance test: a tool to assess the reflective clinician. Teaching and learning in medicine, 2000. 12(4): p. 189-195.

33. Hrynchak, P., S. Glover Takahashi, and M. Nayer, Key-feature questions for assessment of clinical reasoning: a literature review. Medical education, 2014. 48(9): p. 870-883.

34. Page, G. and G. Bordage, The Medical Council of Canada's key features project: a more valid written examination of clinical decision-making skills. Academic medicine: journal of the Association of American Medical Colleges, 1995. 70(2): p. 104-110.

35. Schuwirth, L., et al., Computerized case-based testing: a modern method to assess clinical decision making. Medical Teacher, 1996. 18(4): p. 294-299.

36. Fournier, J.P., A. Demeester, and B. Charlin, Script concordance tests: guidelines for construction. BMC medical informatics and decision making, 2008. 8(1): p. 18.

37. Lubarsky, S., et al., Script concordance testing: From theory to practice: AMEE Guide No. 75. Medical teacher, 2013. 35(3): p. 184-193.

38. Yusoff, M.S.B., ABC of Content Validation and Content Validity Index Calculation. Education in Medicine Journal, 2019. 11(2).

39. Gagnon, R., et al., Script concordance testing: more cases or more questions? Advances in health sciences education, 2009. 14(3): p. 367.

40. Pintrich, P.R., et al., Motivated strategies for learning questionnaire. 1991: Regents of the University of Michigan. 
41. Duncan, T.G. and W.J. McKeachie, The making of the motivated strategies for learning questionnaire. Educational psychologist, 2005. 40(2): p. 117-128.

42. Shamim, M.S., et al., Innovation in ethics and professionalism course: Early experience with portfolioworkbook. JPMA. The Journal of the Pakistan Medical Association, 2016. 66(9): p. 1149-1153.

43. Kember, D. and D.Y. Leung, Establishing the validity and reliability of course evaluation questionnaires. Assessment \& Evaluation in Higher Education, 2008. 33(4): p. 341-353.

44. Pintrich, P.R., et al., Reliability and predictive validity of the Motivated Strategies for Learning Questionnaire (MSLQ). Educational and psychological measurement, 1993. 53(3): p. 801-813.

45. Chandratilake, M., S. McAleer, and J. Gibson, Cultural similarities and differences in medical professionalism: a multi-region study. Med Educ, 2012. 46(3): p. 257-66.

46. Shamim, M.S., et al., Review of ethics teaching in undergraduate medical education, in JPMA. The Journal of the Pakistan Medical Association. 2020. p. 1056-1062.

47. Ahmed, A.A., et al., International medical graduates in the US physician workforce and graduate medical education: current and historical trends. Journal of graduate medical education, 2018. 10(2): p. 214-218.

48. Douaiher, J., D.J. Inciarte, and E. Silva, The intersection of national immigration and healthcare policy. The Journal of the American Board of Family Medicine, 2018. 31(1): p. 163-165.

49. Moazam, F., Reading Caplan in Karachi. Indian journal of medical ethics, 2018. 3(1): p. 66.

50. Hossain, N., Teaching bioethics to postgraduate students in a public sector university: A report from Karachi, Pakistan. Indian journal of medical ethics, 2019. 4(3): p. 242.

51. Al-Eraky, M.M. and H.F. Marei, Medical professionalism: When in Rome,... Romans should help visitors Medical professionalism. 2016.

52. Jafarey, A.M., A degree in bioethics: an" introspective" analysis from Pakistan. 2014.

53. Umran Al-Umran, K., et al., Medical ethics and tomorrow's physicians: an aspect of coverage in the formal curriculum. Medical teacher, 2006. 28(2): p. 182-184.

54. Karagiorgi, Y. and L. Symeou, Translating constructivism into instructional design: Potential and limitations. Journal of Educational Technology \& Society, 2005. 8(1): p. 17-27.

55. Bednar, A.K., et al., Theory into practice: How do we link. Constructivism and the technology of instruction: A conversation, 1992. 8(1): p. 17-34.

56. Dong, H., et al., Twelve tips for using clinical cases to teach medical ethics Twelve tips for using clinical cases to teach medical ethics.

57. Parker, L., L. Watts, and H. Scicluna, Clinical ethics ward rounds: building on the core curriculum. Journal of medical ethics, 2012. 38(8): p. 501-505.

58. van den Bemt, V., et al., Teaching ethics when working with geocoded data: a novel experiential learning approach. Journal of Geography in Higher Education, 2018. 42(2): p. 293-310.

59. Ludwig, S., et al., How can we teach medical students to choose wisely? A randomised controlled cross-over study of video-versus text-based case scenarios. BMC medicine, 2018. 16(1): p. 107. 
60. Braunack-Mayer, A.J., et al., An ethics core curriculum for Australasian medical Schools. The Medical Journal of Australia, 2001. 175(4): p. 205-210.

61. Qian, Y., et al., Insights into medical humanities education in China and the West. Journal of International Medical Research, 2018. 46(9): p. 3507-3517.

62. Saltzburg, L., Is the current state of medical ethics education having an impact on medical students? Online Journal of Health Ethics, 2014. 10(2): p. 2.

63. Pauls, M.A., Teaching and evaluation of ethics and professionalism: in Canadian family medicine residency programs. Canadian Family Physician, 2012. 58(12): p. e751-e756.

64. Cooke, S., J.-f. Lemay, and T. Beran, Evolutions in clinical reasoning assessment: The Evolving Script Concordance Test Evolutions in clinical reasoning assessment: The Evolving Script. 2017.

\section{Figures}

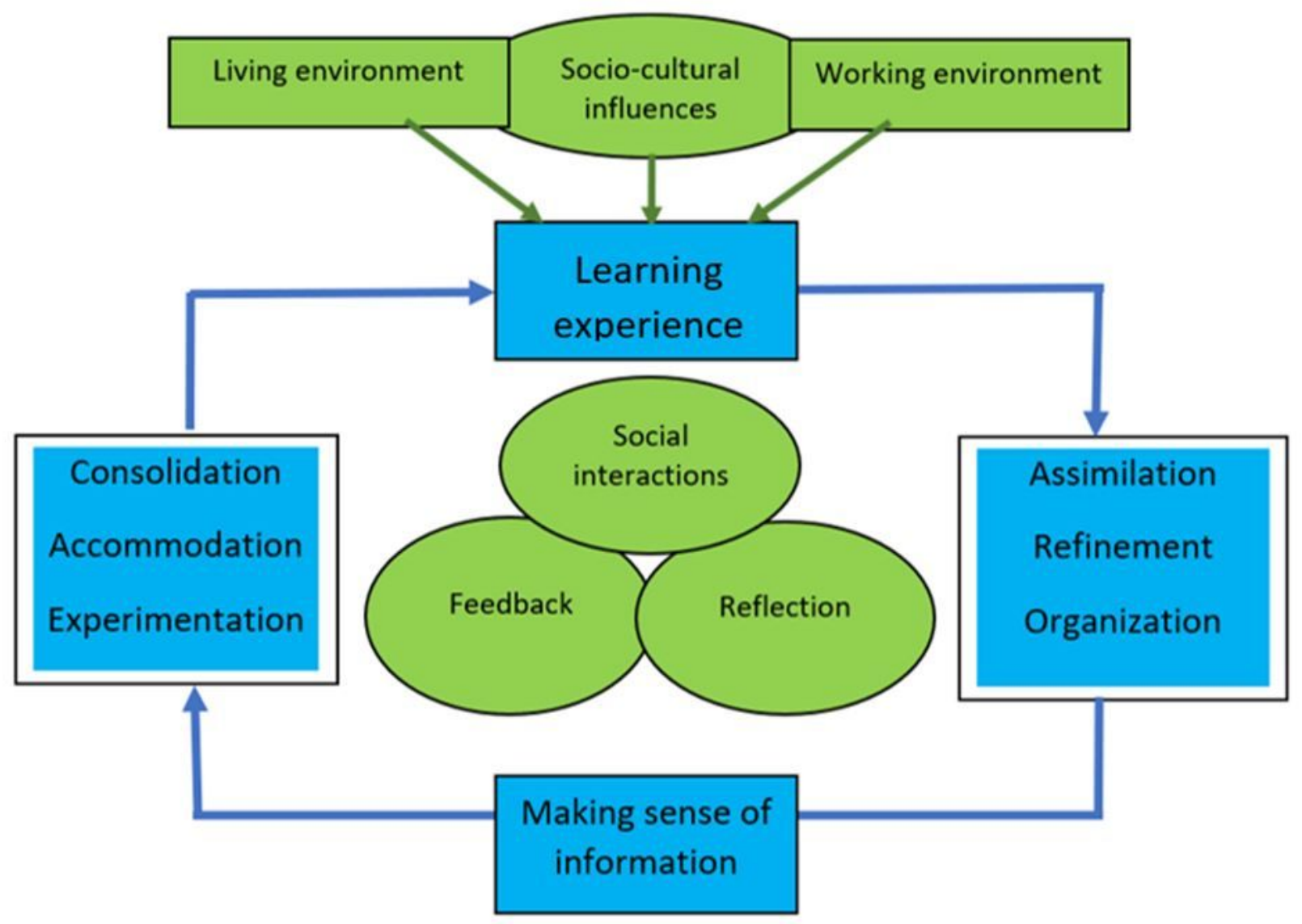

\section{Figure 1}

Contextually Relevant Ethics Education Model, a framework for developing WBEL [18] 


\begin{tabular}{|c|c|}
\hline Table of Content & $\begin{array}{l}\text { Page } \\
\text { Number }\end{array}$ \\
\hline Introduction to the course & 4 \\
\hline Outcomes, Teaching/ Learning Strategies and Assessment & 5 \\
\hline Learning through Portfolio & 8 \\
\hline Ethics \& Professionalism & 10 \\
\hline Principles of Ethics & 11 \\
\hline Duties of a doctor & 12 \\
\hline Medical ethics - Islamic perspective & 14 \\
\hline Communication ethics & 17 \\
\hline Confidentiality & 20 \\
\hline Informed consent & 22 \\
\hline Breaking bad news & 25 \\
\hline Justice and equity & 28 \\
\hline Conflict of interest & 31 \\
\hline Role of doctor in community & 34 \\
\hline End of life care & 36 \\
\hline Medical error & 38 \\
\hline Research ethics & 40 \\
\hline Publication ethics & 42 \\
\hline References & 46 \\
\hline Timetable & 47 \\
\hline
\end{tabular}

\section{Figure 2}

Content topics in The Workbook 


\section{Informed Consent}

Informed consent refers to the principle that every individual has a right to decide for him or herself. The individual should receive the required information to take the decision. In medical practice, a person must give permission before receiving any type of treatment or undergoing investigation (interventional or non-interventional).

For consent to be valid, the person giving consent should have the capacity (emotional and psychological stability) to take decision

The consent it must be:

- Voluntary (without any undue pressure)

- Informed (with knowledge and understanding of the condition, treatment options and possible outcomes)

It is duty of the healthcare professional to ensure that a proper process of acquiring informed consent has taken place before any intervention.

\section{According to the PM\&DC's Code of Ethics 5 :}

"Consent is the 'autonomous authorization of a medical intervention by individual patients.' Patients are entitled to make decisions about their medical care and have the right to be given all available information relevant to such decisions. Patients have the right to refuse treatment and to be given all available information relevant to the refusal."

Guidelines for Helping Patients Reach Informed Consent For acquiring a valid consent, the doctor needs to be sure that patient understands his/her condition and different options for the procedure. Consent can be taken by initiating a conversation, asking questions, and communicating clearly in a way that is understandable for the patient. Following are guidelines for taking an informed consent?:

- Consent process should take place in a relaxed undisturbed atmosphere

- Doctor should carefully describe the diagnosis and possible treatment options in a way that the patient can understand and comprehend

- Avoid medical jargon when describing treatments

- Give patient time to think and ask questions

- Listen carefully to patient's questions and statements

- Use written forms describing procedures and interventions

- Ask your patient to explain the situation to you in their own words

- Be aware of religious or cultural differences that may affect understanding

- Request an interpreter, if necessary

- Document the conversation in the patient's file/charts

JSMU, Karachi 2017

\section{Figure 3}


Ethics Workbook

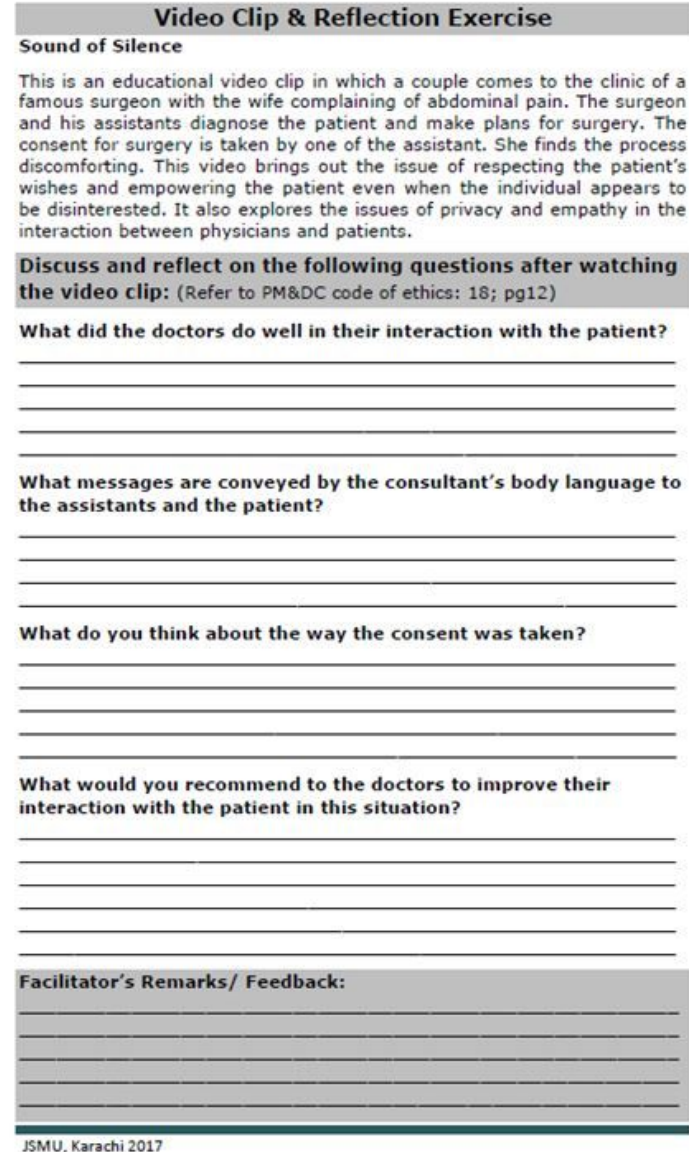

\section{Discuss \& Reflect}

\section{Discuss and answer the following questions}

You are an intern in the hospital. An old friend calls you to ask about the prognosis and treatment options of a patient in your hospital. He says to you: "Can you please check the patients file and find out and let me know about this patient's condition and treatment plan? No one seems to tell the relatives what is going on. They have asked me to find out exactly what is happening". You can easily access the files and all reports.

Can there be any ethical issues in your going through the patient's file and sharing the information with his relatives?

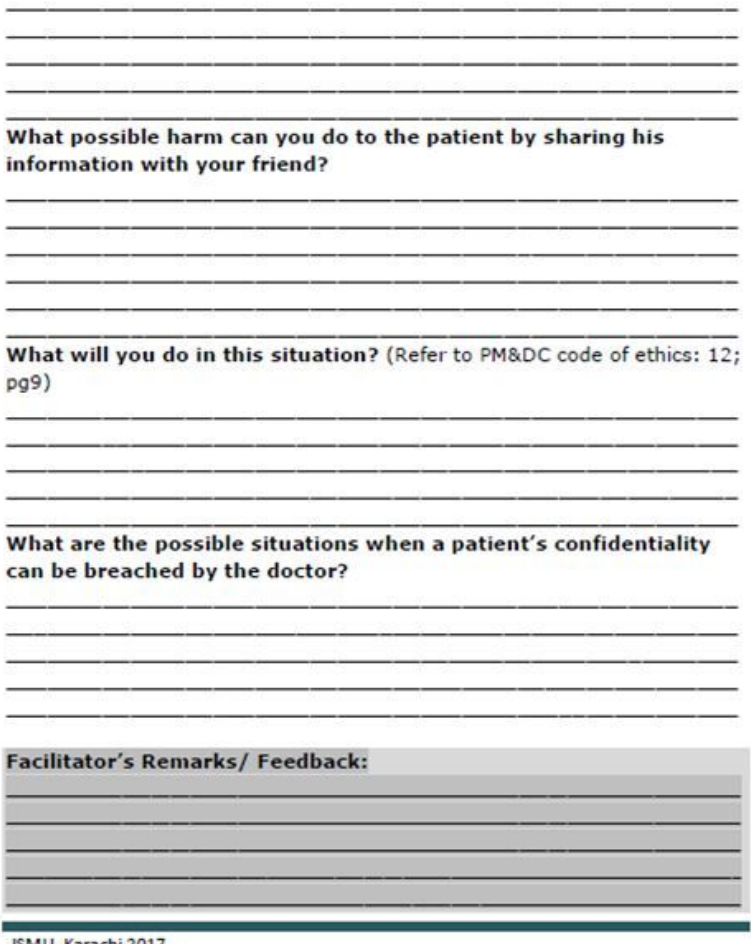

ISMU, Karachi 2017

\section{Figure 4}

Sample learning activities in the Workbook, using case scenario/ video clips with reflective writing and feedback 